\title{
Angiogenesis during primate placentation in health and disease
}

\author{
Christine Wulff ${ }^{1}$, Melanie Weigand ${ }^{1}$, Rolf Kreienberg ${ }^{1}$ and Hamish M. Fraser ${ }^{2}$ \\ ${ }^{1}$ Department of Obstetrics and Gynaecology of the University of Ulm, Prittwitzstraße 43, 89075 Ulm, \\ Germany; and ${ }^{2}$ Medical Research Council, Human Reproductive Sciences Unit, Old Dalkeith Road, \\ Edinburgh EH16 4SB, UK
}

\begin{abstract}
Normal embryonic development is dependent upon a sufficient oxygen, nutrient and waste exchange through the placenta. In primates including humans, this exchange is attained by successful haemochorial placentation which requires the transformation of maternal intramyometrial spiral arterioles by trophoblast invasion to gain uteroplacental circulation, and establishment and maintenance of a competent fetoplacental vasculature. Thus, trophoblast and endothelial cell differentiation, proliferation and invasion occurring during placentation have to be tightly regulated. This review focuses on the diverse developmental steps during haemochorial placentation in humans and other primates and the possible involvement of angiogenic growth factors (vascular endothelial growth factor (VEGF) and angiopoietins (Ang)) in these processes, highlighting the importance of specific actions of angiogenic ligand-receptor pairs. It is hypothesized that VEGF/VEGFR1 and Ang-1/Tie receptor 2 (Tie-2) may regulate trophoblast differentiation and invasion; VEGF/VEGF-R2 and Ang-1/Tie-2 may promote fetoplacental vascular development and stabilization; and Ang-2/Tie-2 may be involved in maternal vascular remodelling. The importance of a tight regulation of angiogenic factors and their endogenous antagonists for normal development of the placenta is demonstrated by failure of this system, resulting in abnormal placenta vascularization and trophoblast invasion associated with intrauterine growth retardation or pre-eclampsia.
\end{abstract}

In recent years, increasing attention has been drawn to the regulation of angiogenesis during placental development. Not only is the placenta a good model in which to study angiogenesis as a result of its high physiological angiogenic activity, but also there is evidence that failure in the development of a functional vasculature leads to severe conditions such as intrauterine growth retardation of the fetus and pre-eclampsia of the mother. Placentation is a dynamic process in which a number of developmental changes occur. Differentiation and developmental processes may be regulated by changes in gene expression of angiogenic growth factors and their receptors. Thus, during placentation the spatial and temporal changes in gene expression may be studied to begin to reveal the physiological role of angiogenic factors in the regulation of trophoblast growth and differentiation and vascular development.

Investigating the processes during placentation is difficult for various reasons. In humans, it is difficult to obtain normal control placentas from intact pregnancies during the second half of gestation. In addition, investigation of the feto-maternal interface and placental bed is

Email: christine.wulff@medizin.uni-ulm.de complicated but necessary when investigating pathological conditions such as pre-eclampsia. Placenta bed biopsies taken during Caesarean sections require some technical expertise and again collection of appropriate controls in humans is nearly impossible. However, interspecies differences during placentation are marked, and it is essential that these differences are considered when extrapolating physiological, endocrinological, immunological or angiogenic data from the animal to the human situation. This review focuses on the regulation of angiogenesis and placental development in primates because of their similarity in developing an efficient placenta for oxygen and nutrient exchange, the haemochorial placenta. Major developmental steps during placentation in primates are the differentiation of the trophoblast, trophoblast invasion of maternal tissue, remodelling of maternal vasculature to gain a uteroplacental circulation and development of a competent fetoplacental vasculature within the trophoblast. Although these processes are common in primates, the placenta of humans is characterized by deep trophoblast invasion and complete remodelling of maternal vasculature by digestion of upper parts of myometrial spiral arterioles. On the basis of knowledge of similarities and differences during placentation in humans and non-human primates, 
it is aimed to piece together the potential role of angiogenic growth factors in molecular regulation of these diverse developmental steps. This is of particular importance when selecting animal models with which the molecular basis of pathological conditions, such as pre-eclampsia, may be investigated. Apart from in humans, natural occurring pre-eclampsia is observed only in few primates such as the patas monkey (Erythrocebus patas) (Palmer et al., 1979), a species that is not widely used in reproduction research. Several attempts have been undertaken to induce pre-eclampsia in various species including non-human primates (Douglas, 1976; Combs et al., 1993; Hennessy et al., 1999); however, all of them have failed to mimic the complete clinical picture of the human disease. The main pathomechanism behind this disorder is believed to be due to incomplete remodelling of maternal spiral arterioles by the invading trophoblast. Only species developing a haemochorial placenta may therefore be affected. Thus, it is of major importance to elucidate physiological and pathological gene expression of angiogenic factors potentially involved in trophoblast growth, invasion and maternal vascular remodelling during primate placentation.

\section{Comparative placentation in humans and non-human primates}

From a macroscopic point of view, primates develop a discoidal placenta which means that the feto-maternal interaction takes place in a roughly circular area. Whereas most non-human primates have a bi-discoidal placenta, humans develop a mono-discoidal placenta. In most primates, for example rhesus monkeys, baboons and marmosets, implantation is superficial (Fig. 1a). The blastocyst is simply attached to the uterine epithelium and the conceptus remains within the uterine lumen (Hearn, 1986). It is only in great apes and humans that the blastocyst becomes completely embedded in the endometrium (Fig. 1a), so that interstitial implantation takes place (Hearn, 1986). The simplest form of the fetomaternal interdigitation found in non-primates is the folded trophoblast type. The uterine surface is thrown into numerous undulations. The folding serves to build a high surface area for the exchanges taking place between fetus and mother. By interruption of the parallel folds and formation of arrays of broad palmate branches an even higher surface is created. The so-called trabecularshaped trophoblast type (Fig. 1b) is the feature of primates (Kaufmann and Burton, 1994). The most efficient way for nutrients and oxygen exchange per volume tissue is to increase the surface area of the trophoblast. This is guaranteed by the formation of a tree-like trophoblast type in which trophoblast villi are formed by repeated branching of stem villi into more slender units. This villous-type placenta (Fig. 1c) is found in humans (Kaufmann and Burton, 1994) and in patas monkeys
(Palmer et al., 1979). With respect to blood supply, in most species the trophoblast is simply apposed to the uterine epithelium without any or minimal destruction of the maternal tissue (epitheliochorial or endotheliochorial implantation, respectively). Thus, there is no direct contact of maternal blood with fetal tissue. Further invasion leads to erosion of maternal vessels, so that the trophoblast is bathed directly by the mother's blood (Fig. 1d). This is termed haemochorial placentation, and is typical for most non-human primates and humans. Here, the barrier between the fetal and mother's blood is very thin, making the exchange of oxygen and nutrients very efficient. A haemomonochorial placenta type develops throughout pregnancy in which the trophoblast consists of the fetal capillaries, interstitial tissue and a monolayer of the syncytiotrophoblast. The depth of trophoblast invasion and maternal vascular remodelling varies among primates; the deepest invasion of the trophoblast with a nearly complete digestion of maternal vessels is found in humans.

\section{Placental development}

Although the time span of the placentation period differs among primates, the fundamental developmental steps necessary for successful placentation are similar: (1) trophoblast invasion; (2) vascularization of the trophoblast to establish and maintain a fetoplacental vasculature; and (3) subsequent maternal vascular remodelling to gain a uteroplacental circulation.

During early placentation the trophoblast differentiates into two compartments, the syncytiotrophoblast and the cytotrophoblast (Fig. 2a). The syncytiotrophoblast invades maternal tissue (Fig. 2b), the depth of invasion depending on the species. Further development leads to penetration of cytotrophoblastic cones into the syncytiotrophoblastic mass (Fig. 2c). First lacunae develop within the syncytiotrophoblast. Further growth and differentiation processes lead to branching of the trophoblast villi and shaping of a placental labyrinth (Fig. 2d). From the extra-embryonic mesenchyme, mesenchymal cores differentiate and grow into the centre of the cytotrophoblast cores. Then endothelial cells differentiate from mesenchymal cells forming the first capillaries of the fetal placenta vasculature. At the same time the beginning of maternal vascular remodelling takes place. By opening of maternal vessels, the placenta labyrinth is filled with maternal blood, flowing around the villous tree. Thus, the haemochorial placenta has developed.

The trophoblast is intrinsically an avascular tissue and so for it to take part in materno-fetal exchange it must develop a functional circulation. Two processes, vasculogenesis and angiogenesis, are involved. Vasculogenesis is defined as the development of new blood vessels from primitive precursor cells, whereas angiogenesis is the development of new vessels from a pre-existing vasculature. Thus, vasculogenesis and angiogenesis are 


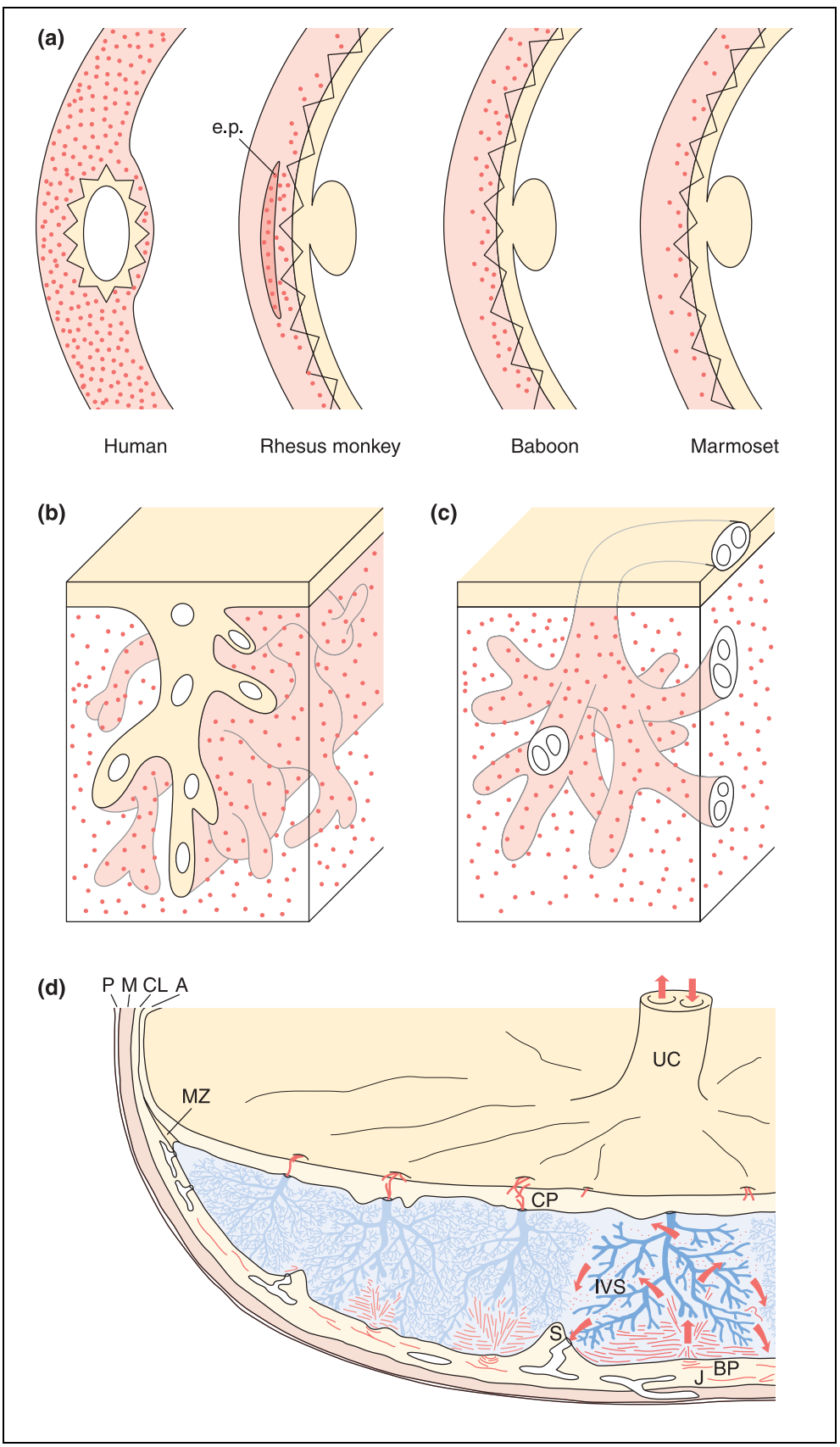

Fig. 1. The differences during placentation between primates and humans. (a) Different implantation types. The human trophoblast is completely embedded into maternal tissue, thus interstitial implantation takes place. In other primates, for example rhesus monkeys, baboons and marmosets, implantation is superficial, but the trophoblast gets into contact with maternal vessels. e.p.: epithelial reaction; $\angle$ : trophoblast. (b) The trabecular trophoblast type typical for non-human primates. (c) The villous trophoblast type found in humans. (d) The mature human placenta illustrating the haemochorial placenta type in which loose centres of villous trees are arranged around maternal arterial inflow areas. Thus, the trophoblast is bathed by the mother's blood (arrows). A: amnion; BP: basal plate; CL: chorion laeve; CP: chorion plate; IVS: intervillous space; J: junctional zone; $M$ : myometrium; $M Z$ : marginal zone between placenta and fetal membranes; P: perimetrium; S: septum; UC: umbilical cord. (a) Redrawn from Hearn (1986). (b,c,d) Redrawn from Kaufmann and Burton (1994). 


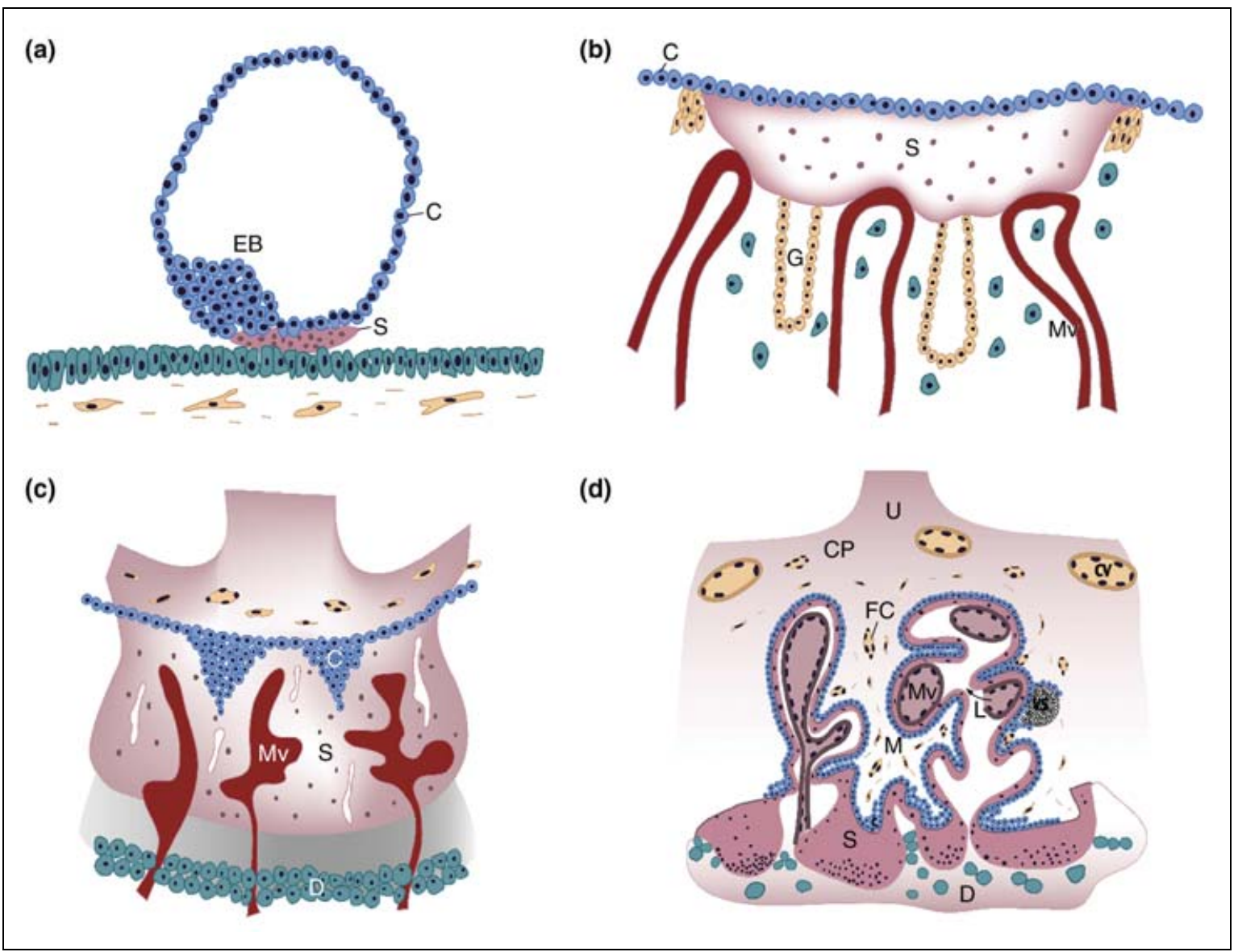

Fig. 2. Early placenta development in primates. (a) During implantation the trophoblast differentiates into the syncytiotrophoblast (S) and the cytotrophoblast (C). EB: embryoblast. (b) The syncytiotrophoblast begins to invade maternal tissue, making contact with maternal vessels (Mv). G: uterine glands. (c) After deeply penetrating maternal tissue, first lacunae develop within the syncytiotrophoblast and cytotrophoblastic cones grow into the syncytiotrophoblast mass. First fetal capillaries appear within the chorion plate. D: decidua. (d) Further development leads to branching of the trophoblast, resulting in a trabecular tree. The trabecle contain mesenchyme (M), fetal capillaries (FC) and a single layer of cytotrophoblast cells. The barrier to the labyrinth (L) is built by a thin syncytiotrophoblast layer. Maternal vessels are opened (arrow) so that the placental labyrinth is filled with maternal blood. By this time the haemochorial placenta has developed. CP: chorion plate; U: umbilical cord.

both involved in fetal vascular development. Conversion of maternal spiral arterioles associated with angiogenesis differs between non-human primates and humans. In humans, the trophoblast invades deep into the maternal uterine tissue causing complete digestion of the upper parts of maternal spiral arterioles. By the open endings of maternal vessels, maternal blood is released into the placenta labyrinth and flows around the trophoblast villi and is drained by venous sinosoids. A different situation occurs in non-human primates, for example in marmosets, in which the invasion of maternal tissue is less deep. Here, maternal vessels persist throughout pregnancy. However, gaps in the maternal vascular wall are found in increasing number throughout pregnancy, from which the placental labyrinth is filled with maternal blood (Fig. 2d).

\section{Principal molecular regulators of angiogenesis}

Co-ordination of the diverse developmental steps outlined above appears to be mediated by locally acting growth factors, principally placenta growth factor (PIGF), vascular endothelial growth factor (VEGF) and angiopoietins (Ang-1 and Ang-2). These factors appear not only to regulate vascular development and remodelling during placentation but also act as growth factors for driving growth and differentiation processes such as trophoblast invasion and maturation. Both growth factor families act via their tyrosine kinase receptors (Hanahan, 1997). PIGF binds only to the VEGF-R1 receptor, whereas VEGF binds to the VEGF-R1 and VEGF-R2 receptors (Gale and Yancopoulos, 1999). The physiological role of PIGF in placental development is at present unclear. 
PIGF-deficient mice do not show any signs of abnormality during development, reproduction or post-natal life (Charnock-Jones and Burton, 2000).

VEGF activation of the VEGF-R2 receptor causes endothelial cell differentiation and proliferation (Bernatchez et al., 1999), whereas binding to the VEGF-R1 receptor mediates endothelial cell interaction and tubule formation (Fong et al., 1995). Tie receptors (Tie-1 and Tie-2) constitute additional tyrosine kinases involved in vascular remodelling. Tie-1 is present on the trophoblast (Vuorela et al., 2000), but the ligand for this receptor and its function during placentation is unknown. The Tie-2 receptor is used by the angiopoietins. Ang- 1 activation of Tie-2 receptor stabilizes newly formed vessels by recruiting perivascular support cells such as pericytes (Suri et al., 1996). Ang-2 is a competitive antagonist to Ang-1 at the Tie-2 receptor, which may cause destabilization of the vasculature (Maisonpierre et al., 1997). This destabilization will facilitate the actions of angiogenic inducers such as VEGF but may destroy newly formed vessels when VEGF is reduced.

Thus, VEGF mediates vasculogenesis, whereas angiopoietins are involved in the processes of vascular remodelling during angiogenesis. It is proposed that VEGF drives fetoplacental vascular development, whereas the angiopoietins are associated with reworking of the maternal vessels during placentation. The angiogenic factors are expressed during early placenta development before fetal vascular development or maternal vascular remodelling is induced (Ghosh et al., 2000; Wulff et al., 2002a; Rowe et al., 2003). Here, VEGF and angiopoietins may also act as growth factors to promote trophoblast growth and migration.

\section{Trophoblast invasion, fetoplacental vascular development and maternal vascular remodelling during haemochorial placentation}

The current literature on the localization of angiogenic growth factors in the placenta is summarized here. There are no functional studies in primates demonstrating how placentation is regulated on a molecular basis by these factors. However, by piecing together the current knowledge of angiogenic factor expression, a picture of possible molecular regulatory processes emerges.

For all primates studied, including humans, VEGF and its receptors have been localized in the placenta during early pregnancy at the time when the syncytiotrophoblast penetrates maternal tissue before vascular development and remodelling takes place. VEGF is expressed in maternal decidual cells (Charnock-Jones et al., 1994; Cooper et al., 1995; Wulff et al., 2002a) and in the invading trophoblast (Charnock-Jones et al., 1994; Geva et al., 2002). At that time, of the two VEGF receptors only VEGF-R1 is expressed in the primate placenta, and its expression is localized in the invading extravillous trophoblast (Charnock-Jones et al., 1994; Clark et al., 1996; Wulff et al., 2002a). These observations may imply that VEGF binding to VEGF-R1 receptor is involved in driving trophoblast invasion.

Within the trophoblast tree, VEGF is localized in the cytotrophoblast cells (Geva et al., 2002; Wulff et al., 2002a; Zhou et al., 2002) and VEGF-R1 is localized in the villous trophoblast (Sharkey et al., 1993; Hildebrandt et al., 2001; Geva et al., 2002; Wulff et al., 2002a; Zhou et al., 2002), indicating that VEGF/VEGF-R1 may play an important role in the co-ordination of trophoblast differentiation and migration in addition to regulating trophoblast invasion.

In addition, the angiopoietins are expressed during the very early placentation period in primates (Rowe et al., 2003), indicating their involvement in the regulation of trophoblast growth. In monkeys, Ang-1 is highly expressed in the syncytiotrophoblast, whereas its receptor, Tie-2, is located in the cytotrophoblast (Geva et al., 2002; Wulff et al., 2002a). Similar observations have been made in the human placenta, in which Ang-1 and Tie-2 have also been detected in the cytotrophoblastsyncytiotrophoblast bilayer (Dunk et al., 2000; Geva et al., 2002). It was demonstrated by using a recombinant Tie-2-Fc (a construct containing the extracellular domain of the Tie-2 receptor fused to the Fc portion of human IgG) to inhibit Ang-1 action, that Ang-1 stimulated trophoblast growth and migration in vitro (Dunk et al., 2000). In marmosets, increasing gene expression of Ang-1 was found during gestation (Wulff et al., 2002a). Thus, Ang-1 may trigger the in-growth of the cytotrophoblast cones in the syncytiotrophoblast, whereas the relatively higher expression later during placentation may be required for branching of the villi and shaping the labyrinth.

When the trophoblast tree develops, the fetoplacental vascular system differentiates simultaneously, including endothelial cell differentiation, proliferation, tubulus formation and stabilization. In the majority of reports, the VEGF-R2 receptor was found to be exclusively expressed on endothelial cells or mesenchymal cells from which endothelial cells differentiate (Clark et al., 1996; Helske et al., 2001; Hildebrandt et al., 2001; Geva et al., 2002; Wulff et al., 2002a), whereas VEGF expression was localized in the trophoblast, indicating that endothelial cell differentiation, migration and proliferation as the essential steps for building the primary vascular network are mediated by a VEGFNEGF-R2 ligand-receptor pair in a paracrine system.

Whereas fetal capillaries of the trophoblast are thinwalled in order to allow oxygen diffusion, chorion vessels are stabilized by a thick wall of pericytes, smooth muscle cells or both to fulfil their task of collection and draining all fetal placental blood. There are few studies demonstrating Tie-2 expression in the placenta (Dunk et al., 2000; Goldman-Wohl et al., 2000; Wulff et al., 2002a). Tie-2 was found to be highly expressed in the wall of chorion vessels, whereas in fetal capillaries 
(a)

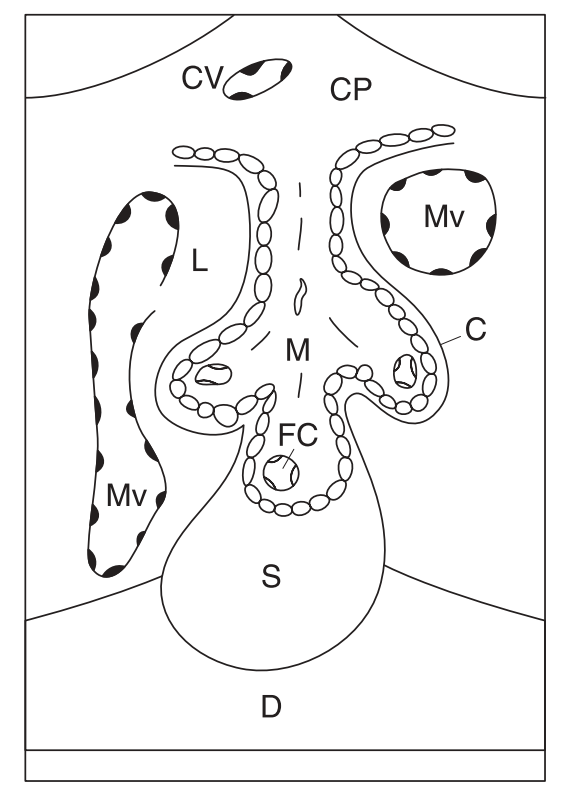

(b)

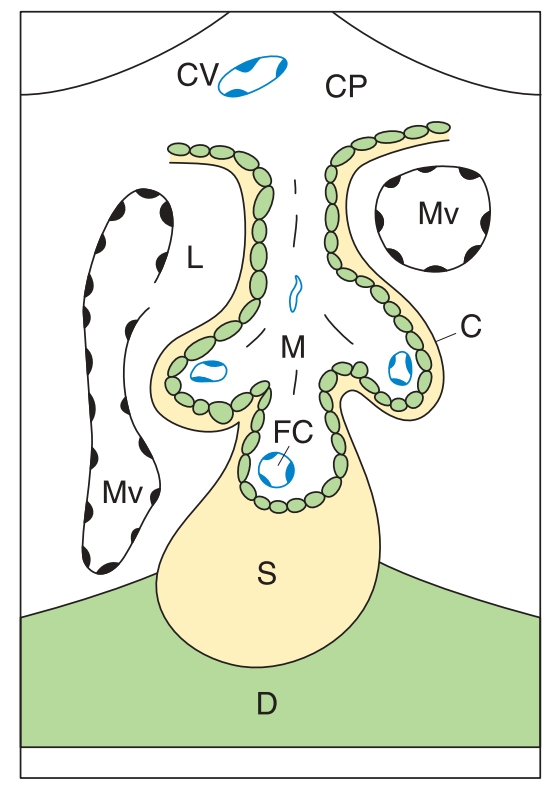

(c)

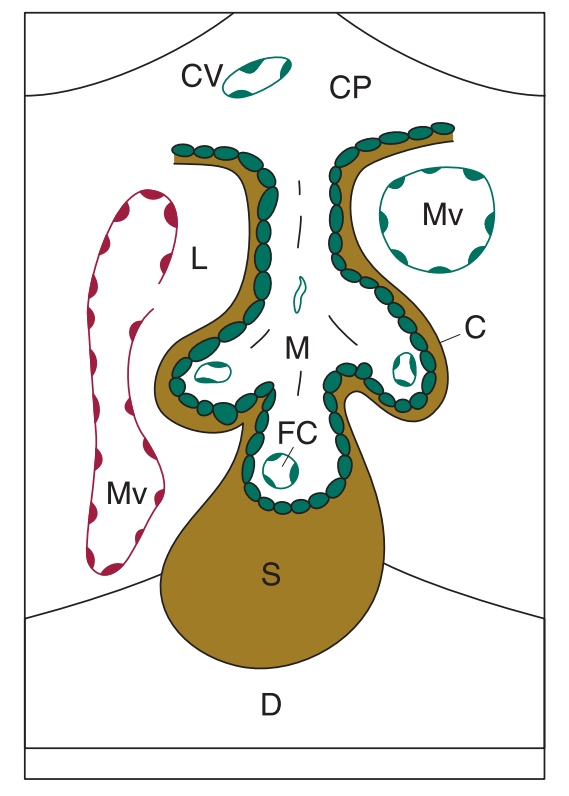

Fig. 3. (a) Schematic diagram of a cross-section of the marmoset placenta. (b) Vascular endothelial growth factor (VEGF)/VEGFR1/VEGF-R2 expression patterns. VEGF (green) was expressed in the maternal decidua (D) and in the cytotrophoblast cells (C). VEGF-R1 (yellow) was expressed in the invading syncytiotrophoblast (S) and the syncytiotrophoblast lining the trabecula. VEGF-R2 (blue) was localized to fetal capillaries (FC) and chorion vessels (CV). (c) Ang-1/Ang-2/Tie-2 expression patterns. Ang-1 (brown) was focally expressed in the syncytiotrophoblast. Ang-2 (red) was exclusively localized in the endothelium of maternal vessels (Mv). The Tie-2 receptor (green) was found in parts of the cytotrophoblast, in the maternal endothelium, in fetal capillaries and in the wall of chorion vessels. CP: chorion plate; L: labyrinth; M: mesenchyme.

only low expression of Tie-2 was observed (Wulff et al., 2002a). These results may imply that here the Ang-1/ Tie-2 ligand-receptor pair acts on the fetal vasculature, especially on chorion vessels in order to induce stability. In humans, Ang-1 expression was found in the media of stem villous vessels at term (Dunk et al., 2000), which is also consistent with the reported role in vessel maturation and stabilization.

Maternal vessels have to be transformed to attain uteroplacental circulation. In humans, the invasion process of the trophoblast is so deep that upper parts of maternal spiral arterioles become completely digested and the placental labyrinth is filled by the open endings of the spiral arterioles. In humans, Ang-2 is localized to the syncytiotrophoblast and Tie-2 is localized to the maternal endothelium (Dunk et al., 2000; Zhang et al., 2001), indicating a paracrine mechanism during maternal remodelling (Goldman-Wohl et al., 2000). A different situation occurs in marmosets for example, in which the invasion process is less deep and maternal vessels persist throughout pregnancy. The maternal vessels become opened, forming gaps through which maternal blood is released into the labyrinth (Merker et al., 1988). In marmosets, a unique pattern of Ang-2 expression was discovered, the mRNA being exclusively localized in maternal endothelial cells together with its receptor
Tie-2 (Wulff et al., 2002a). High expression of Ang2 mRNA was detected during early gestation, when maternal remodelling mainly takes place. Ang-2 may be a possible candidate for induction of maternal vascular transformation because it destabilizes the vasculature (Maisonpierre et al., 1997). In marmosets this destabilization process is a local event, which may be driven by Ang-2/Tie-2. In humans, although the remodelling process of maternal vessels may be supported by Ang$2 / \mathrm{Tie}-2$, it is mainly driven by the aggressive trophoblast invasion.

In summary, for humans and other primates it is evident that processes involved in haemochorial placentation are supported by angiogenic growth factors. The most comprehensive study investigating both growth factor families, VEGF and angiopoietins, and their receptors during early mid- and late pregnancy has been undertaken in marmoset monkeys, and the study indicates a tight spatial and temporal regulation of diverse developmental processes during placentation by angiogenic inducers (Wulff et al., 2002a; Rowe et al., 2003). The results are summarized (Fig. 3). In accordance with the above reports it was specifically hypothesized that VEGF/EGF-R1 and Ang1/Tie-2 may be involved in trophoblast differentiation and invasion; VEGF/VEGFR2 and Ang-1/Tie-2 may trigger fetoplacental vascular 
development, whereas Ang-2/Tie-2 may support the remodelling processes of the maternal vasculature.

\section{Clinical aspects}

Normal development of the embryo in primates is dependent on successful haemochorial placentation resulting in differentiation of an organ responsible for oxygen, nutrients and waste exchange. The haemochorial placenta is found in humans, showing interstitial implantation, deepest trophoblast invasion, villous tree formation and nearly complete digestion of maternal spiral arterioles, compared with the placentas of other primates, and provides the most effective oxygen exchange per volume of tissue. On the other hand, the system may be more vulnerable to disturbance during normal placentation resulting in pathological conditions for the embryo (intrauterine growth retardation) or for the mother (pre-eclampsia or haemolysis, elevated liver enzyme activity, and low platelets syndrome (HELLP syndrome). Thus, these conditions are rarely observed in other primates. In the literature, symptoms of spontaneously occurring pre-eclampsia are reported, for example in patas monkeys (Palmer et al., 1979). As in humans, patas monkeys also develop a villous trophoblast tree. Although the pathophysiology of preeclampsia is still unknown, the hypothesis of dysregulated endovascular trophoblast invasion as a key step for the development of pre-eclampsia is favoured (Kaufmann et al., 2003).

When discussing possible contributions of angiogenic factors in the pathogenesis of the above-mentioned disorders, it must be borne in mind that anything studied may be changed without knowing if it is a cause or consequence of the alterations. However, there is evidence that angiogenic factors may contribute to pregnancy-associated diseases such as pre-eclampsia. Pre-eclampsia is characterized by hypertension, proteinuria and oedema and affects $5 \%$ of pregnancies. In severe cases, pre-eclampsia leads onto an eclamptic episode. For development of the syndrome a cascade of events has to take place in which functional alterations in the maternal vasculature may play a key role (Roberts et al., 1989). In pre-eclamptic placentas the extent of interstitial trophoblast invasion is rudimentary (Roberts, 1998), and maternal vessels containing cytotrophoblasts are difficult to detect (Zhou et al., 1997). The initial event causing pre-eclampsia is thought to be abnormal trophoblast invasion of spiral arterioles (Granger et al., 2001). Reduced maternal vessel conversion is generally believed to culminate in reduced uteroplacental circulation resulting in relative placental bed hypoxia or ischaemia. This may lead to a widespread activation and dysfunction of the maternal vascular endothelium, resulting in appearance of symptoms associated with preeclampsia (Granger et al., 2001). As described above, angiogenic factors may drive invasion processes of the trophoblast during pregnancy. Accordingly, during pregnancies complicated by conditions such as preeclampsia and HELLP syndrome, in which trophoblast invasion is disturbed, levels of VEGF, PIGF, VEGF-R1 and Ang-2 are decreased (Torry et al., 1998; Reuvekamp et al., 1999; Zhang et al., 2001; Zhou et al., 2002). Conversely, hypoxia may induce local pseudovasculogenesis as also associated with pre-eclampsia by local over-expression of angiogenic factors such as VEGF or angiopoietins. This contention is supported by culture studies of villous explants, in which a reduction of oxygen tension significantly raised the expression of Ang2 mRNA (Zhang et al., 2001). Furthermore, it has been demonstrated that VEGF may be induced by hypoxia (Popovici et al., 1999; Rossler et al., 1999; Ahmed et al., 2000). A role for factors with anti-angiogenic actions which may balance the physiological angiogenic activity was postulated to be of pathophysiological importance for impaired trophoblast and vascular development during pre-eclampsia (Maynard et al., 2003). It has been demonstrated that the receptor for PIGF and VEGF is present in a soluble form (sVEGF-R1) (He et al., 1999), which is expressed in human (Clark et al., 1998) and non-human primate placentas (Wulff et al., 2002a). The VEGF-R1 pre-mRNA can be alternatively spliced, resulting in the membrane-bound VEGF-R1 receptor and sVEGF-R1 comprising the ligand-binding domain of VEGF-R1 receptor (He et al., 1999), thus becoming a potent endogenous antagonist for VEGF and PIGF (Clark et al., 1998). sVEGF-R1 as an endogenous antagonist of VEGF and PIGF is increased during pre-eclampsia (Helske et al., 2001; Zhou et al., 2002; Maynard et al., 2003). Maynard et al. (2003) demonstrated that sVEGFR1 upregulation, presumably from the placenta, is also associated with increased systemic levels of sVEGFR1. Furthermore, free VEGF and PIGF were decreased. Thus, a lack of serum PIGF and VEGF may cause the systemic maternal endothelial defect which is reflected by the symptoms of pre-eclampsia. In summary, imbalance of angiogenic factors and their endogenous antagonists may contribute to the pathogenesis of preeclampsia.

\section{Animal models in clinical research}

Owing to methodological limitations of studying preeclampsia in humans, a variety of animal models has been investigated (Douglas, 1976; Combs et al., 1993; Hennessy et al., 1999; Podjarny et al., 1999). Preeclampsia was induced by reducing uteroplacental perfusion using an aortic clip technique or chronic nitric oxide synthase inhibition. These models succeeded in mimicking some of the symptoms of pre-eclampsia, but failed to induce the complete clinical syndrome. One reason might be that they failed to inhibit the primary step of the condition, which appears to be inhibition of trophoblast invasion and maternal vascular remodelling. 
As outlined above, angiogenic factors may play a crucial role in the regulation of these events. However, conclusive functional data are lacking and experiments designed to inhibit angiogenesis selectively during early pregnancy in suitable animal models are required. In rats, administration of recombinant adenovirus encoding sVEGF-R1 gene on day 8 of pregnancy induced hypertension and albuminuria (Maynard et al., 2003). Thus, inhibition of angiogenic factors during early pregnancy may be the key for simulating pre-eclampsia. It has recently been demonstrated that selective inhibition of angiogenic factors, such as VEGF, can be achieved in primates by administering specific antagonists and that physiological angiogenesis in the reproductive tract can be successfully inhibited (Wulff et al., 2001a,b, 2002b; Fraser et al., 2002). Thus, there is now the potential for designing non-human primate studies in which angiogenic factors can be manipulated in early pregnancy in order to dissect their role in intrauterine growth retardation and pre-eclampsia.

\section{Concluding remarks}

It appears that during normal haemochorial placentation, the processes of trophoblast invasion, fetoplacental vascular development and maternal vascular remodelling, VEGF, angiopoietins and their receptors may be involved in the regulation of these processes. Despite minor developmental differences during placentation between non-human primates and humans, it is proposed that VEGF/VEGF-R1 and Ang-1/Tie-2 may play a role in trophoblast growth and invasion. VEGF/VEGF-R2 and Ang-1/Tie-2 may regulate fetoplacental vascular development and Ang-2/Tie-2 may support maternal vascular remodelling. Furthermore, the importance of a balanced system of the spatial and temporal expression of these angiogenic factors and their endogenous antagonists for normal placentation is demonstrated by comparison of changes in the expression patterns of these factors in severe compromised pregnancies (pre-eclampsia and intrauterine growth retardation). The clinical condition of pre-eclampsia caused by insufficient trophoblast invasion and maternal vascular remodelling is observed only in primates that develop a haemochorial, villous placenta. As angiogenic factors may be crucial in regulating these events and angiogenesis has been successfully inhibited in primates by targeting specific angiogenic factors, it is hypothesized that a primate model for pre-eclampsia may be established by local treatment of the uteroplacental system with angiogenic factor antagonists during early placentation.

\section{References}

Key references are identified by asterisks.

*Ahmed A, Dunk C, Ahmed S and Khaliq A (2000) Regulation of placental vascular endothelial growth factor (VEGF) and placenta growth factor
(PIGF) and soluble Flt-1 by oxygen - a review Placenta 21 Supplement A $16-24$

Bernatchez PN, Soker S and Sirois MG (1999) Vascular endothelial growth factor effect on endothelial cell proliferation, migration, and plateletactivating factor synthesis is Flk-1-dependent Journal of Biological Chemistry 27431 047-31 054

*Charnock-Jones DS and Burton GJ (2000) Placental vascular morphogenesis Baillieres Best Practice and Research Clinical Obstetrics and Gynaecology 14 953-968

Charnock-Jones DS, Sharkey AM, Boocock CA, Ahmed A, Plevin R, Ferrara N and Smith SK (1994) Vascular endothelial growth factor receptor localization and activation in human trophoblast and choriocarcinoma cells Biology of Reproduction $\mathbf{5 1}$ 524-530

Clark DE, Smith SK, Sharkey AM and Charnock-Jones DS (1996) Localization of VEGF and expression of its receptors flt and KDR in human placenta throughout pregnancy Human Reproduction 11 10901098

Clark DE, Smith SK, He Y, Day KA, Licence DR, Corps AN, Lammoglia R and Charnock-Jones DS (1998) A vascular endothelial growth factor antagonist is produced by the human placenta and released into the maternal circulation Biology of Reproduction 59 1540-1548

Combs Ca, Katz MA, Kitzmiller JL and Brescia RJ (1993) Experimental preeclampsia produced by chronic constriction of lower aorta: validation with longitudinal blood pressure measurements in conscious rhesus monkey American Journal of Obstetrics and Gynecology 169 215-223

Cooper JC, Sharkey AM, McLaren J, Charnock-Jones DS and Smith SK (1995) Localization of vascular endothelial growth factor and its receptor, flt, in human placenta and decidua by immunohistochemistry Journal of Reproduction and Fertility 105 205-213

Douglas BH (1976) The rat as a model for preeclampsia Perspectives in Nephrology and Hypertension 5 411-419

Dunk C, Shams M, Nijjar S, Rhaman M, Qiu Y, Bussolati B and Ahmed A (2000) Angiopoietin-1 and angiopoietin-2 activate trophoblast Tie-2 to promote growth and migration during placental development American Journal of Pathology 156 2185-2199

Fong GH, Rossant J, Gertsenstein M and Breitman ML (1995) Role of the Flt-1 receptor tyrosine kinase in regulating the assembly of vascular endothelium Nature 376 66-70

Fraser HM, Wulff C, Wiegand SJ and Rudge JS (2002) Inhibition of vascular endothelial growth factor action in the primate reproductive system Biology of Reproduction $\mathbf{6 6}$ Supplement $\mathbf{1} 82$

Gale NW and Yancopoulos GD (1999) Growth factors acting via endothelial cell-specific receptor tyrosine kinases: VEGFs, angoipoietins, and ephrins in vascular development Genes and Development 13 10551066

Geva E, Ginzinger DG, Zaloudek CJ, Moore DH, Byrne A and Jaffe RB (2002) Human placental vascular development: vasculogenic and angiogenic (branching and nonbranching) transformation is regulated by vascular endothelial growth factor-A, angiopoietin-1 and angiopoietin-2 Journal of Clinical Endocrinology and Metabolism 87 4213-4224

Ghosh D, Sharkey AM, Charnock-Jones DS, Dhawan L, Dhara S, Smith SK and Sengupta J (2000) Expression of vascular endothelial growth factor (VEGF) and placental growth factor (PIGF) in conceptus and endometrium during implantation in the rhesus monkey Molecular Human Reproduction 6 935-941

Goldman-Wohl DS, Ariel I, Greenfield C, Lavy Y and Yagel S (2000) Tie-2 and angiopoietin-2 expression at the fetal-maternal interface: a receptor ligand model for vascular remodelling Molecular Human Reproduction 6 81-87

Granger JP, Alexander BT, Llinas MT, Bennett WA and Khalil RA (2001) Pathophysiology of hypertension during preeclampsia linking placental ischemia with endothelial dysfunction Hypertension 38 718-722

Hanahan D (1997) Signaling vascular morphogenesis and maintenance Science 277 48-50

He Y, Smith SK, Day KA, Clark DE, Licence DR and Charnock-Jones DS (1999) Alternative splicing of vascular endothelial growth factor (VEGF)R1 (FLT-1) pre-mRNA is important for the regulation of VEGF activity Molecular Endocrinology 13 537-545 
Hearn JP (1986) The embryo-maternal dialogue during early pregnancy in primates Journal of Reproduction and Fertility $\mathbf{7 6}$ 809-819

Helske S, Vuorela P, Carpen O, Hornig C, Weich $\mathrm{H}$ and Halmesmaki E (2001) Expression of vascular endothelial growth factor receptors 1, 2 and 3 in placentas from normal and complicated pregnancies Molecular Human Reproduction 7 205-210

Hennessy A, Gillin AG, Duggin GG, Horwath JS and Tiller DJ (1999) Low-dose nitro-L-arginine administration in baboon (Papio hamadryas) pregnancy Clinical Experiments in Pharmacology and Physiology 26 849-852

Hildebrandt VA, Babischkin JS, Koos RD, Pepe GJ and Albrecht ED (2001) Developmental regulation of vascular growth/permeability factor messenger ribonucleic acid levels in and vascularization of the villous placenta during baboon pregnancy Endocrinology 142 2050-2057

Kaufmann P and Burton G (1994) Anatomy and genesis of the placenta. In The Physiology of Reproduction 2nd Edn pp 441-484 Eds E Knobil and JD Neill. Raven Press, New York

*Kaufmann P, Black S and Huppertz B (2003) Endovascular trophoblast invasion: implications of the pathogenesis of intrauterine growth retardation and preeclampsia Biology of Reproduction 69 1-7

Maisonpierre PC, Suri C, Jones PF et al. (1997) Angiopoietin-2, a natural antagonist for Tie2 that disrupts in vivo angiogenesis Science 277 55-60

* Maynard SE, Min JJ, Merchan J et al. (2003) Excess placental soluble fmslike tyrosine kinase (sFlt) may contribute to endothelial dysfunction, hypertension, and proteinuria in preeclampsia Journal of Clinical investigation 111 649-658

Merker HJ, Bremer D, Csato W, Heger W and Gossrau R (1988) Development of the marmoset placenta. In Non Human Primates: Developmental Biology and Toxicology pp 245-272 Eds D Neubert, $\mathrm{Hj}$ Merker and AG Hendricks. Ueberreuther Wissenschaft, Berlin

Palmer AE, London WT, Sly DL and Rice JM (1979) Spontaneous preeclamptic toxemia of pregnancy in the pata monkey (Erythorcebus patas) Laboratory Animal Science 29 102-106

Podjamy E, Baylis C and Losonczy G (1999) Animal models of preeclampsia Seminars in Perinatology 23 2-13

Popovici RM, Irwin JC, Giaccia AJ and Giudice LC (1999) Hypoxia and CAMP stimulate vascular endothelial growth factor (VEGF) in human endometrial stromal cells: potential relevance to menstruation and endometrial regeneration Journal of Clinical Endocrinology and Metabolism $842245-2248$

Reuvekamp A, Velsing-Aarts V, Poulina IEJ, Capello JJ and Duits AJ (1999) Selective deficit of angiogenic growth factors characterises pregnancies complicated by preeclampsia British Journal of Obstetrics and Gynaecology 106 1019-1022

Roberts JM (1998) Endothelial dysfunction in preeclampsia Seminars in Reproductive Endocrinology 16 5-15

Roberts JM, Taylor RN, Musci TJ, Rodgers GM, Hubel CA and McLaughlin MK (1989) Preeclampsia: an endothelial cell disorder American Journal of Obstetrics and Gynecology 161 1200-1204
Rossler J, Breit S, Havers W and Schweigerer L (1999) Vascular endothelial growth factor expression in human neuroblastoma: up-regulation by hypoxia International Journal of Cancer 81 113-117

Rowe AJ, Wulff C and Fraser HM (2003) Localisation of vascular endothelial growth factor and angiopoietins during the peri-implantation period and early pregnancy in the marmoset Reproduction 126 227-238

Sharkey AM, Charnock-Jones DS, Boocock CA, Brown KD and Smith SK (1993) Expression of mRNA for vascular endothelial growth factor in human placenta Journal of Reproduction and Fertility 99 609-615

Suri C, Jones P, Patan S, Bartunkova S, Maisonpierre P, Davis S, Sato T and Yancopoulos G (1996) Requisite role of angiopoietin-1, a ligand for the Tie-2 receptor during embryonic angiogenesis Cell 87 11711180

Torry DS, Wang HS, Wang TH, Caudle MR and Torry RJ (1998) Preeclampsia is associated with reduced serum levels of placenta growth factor American Journal of Obstetrics and Gynecology 1791539 1544

Vuorela P, Carpen O, Tulppala M and Halmesmaki E (2000) VEGF, its receptors and the tie receptors in recurrent miscarriage Molecular Human Reproduction 6 276-282

Wulff C, Wiegand SJ, Saunders PTK, Scobie GA and Fraser HM (2001a) Angiogenesis during follicular development and its inhibition by Flt-1FC (VEGF Trap $\left.{ }_{A 40}\right)$ in the primate Endocrinology 142 3244-3254

Wulff C, Wilson H, Rudge JS, Wiegand SJ, Lunn SF and Fraser HM (2001b) Luteal angiogenesis: prevention or intervention by treatment with vascular endothelial growth factor Trap ${ }_{A 40}$ Journal of Clinical Endocrinology and Metabolism 86 3377-3386

Wulff C, Dickson SE, Wilson H, Wiegand SJ and Fraser HM (2002a) Hemochorial placentation in the primate: expression of vascular endothelial growth factor, angiopoietins and their receptors throughout pregnancy Biology of Reproduction 66 802-812

Wulff C, Wilson H, Wiegand SJ, Rudge JS and Fraser HM (2002b) Prevention of thecal angiogenesis, antral follicular growth and ovulation in the primate by treatment with endothelial growth factor trap R1R2 Endocrinology 143 2797-2807

Zhang EG, Smith SK, Baker PN and Charnock-Jones DS (2001) The regulation and localisation of angiopoietin-1,-2 and their receptor Tie2 in normal and pathologic human placentae Molecular Medicine 7624 635

Zhou Y, Damsky CH and Fisher SJ (1997) Preeclampsia is associated with failure of human cytotrophoblasts to mimic a vascular adhesion phenotype. One course of defective endovascular invasion in this syndrome? Journal of Clinical Investigation 99 2152-2164

*Zhou Y, McMaster M, Woo K, Janatpour M, Perry J, Karpanen T, Alitalo K, Damsky C and Fisher SJ (2002) Vascular endothelial growth factor ligands and receptors that regulate human cytotrophoblast survival are dysregulated in severe preeclampsia and hemolysis, elevated liver enzymes and low platelets syndrome American Journal of Pathology $\mathbf{1 6 0}$ 1405-1423 